\title{
Facilitators and barriers to the delivery of school-based smoking prevention interventions for children and young people: a protocol for a systematic review of qualitative studies
}

Fiona Dobbie ${ }^{1 *}$ (1) Kathryn Angus ${ }^{1}$, Hannah Littlecott ${ }^{2}$, Karen Allum', Valerie Wells ${ }^{3}$, Amanda Amos ${ }^{4}$, Sally Haw ${ }^{5}$ and Linda Bauld ${ }^{1}$

\begin{abstract}
Background: Despite a decline in child and adult smoking prevalence, young people who smoke (even occasionally) can rapidly become addicted to nicotine, with most adult smokers initiating smoking before they are 18. Schools have long been a popular setting to deliver youth smoking prevention interventions, but evidence of the effectiveness of school-based prevention programmes is mixed, and outcomes vary by the type of programme delivered. Existing systematic reviews that explore the factors contributing to the success or failure of school-based smoking prevention programmes often exclude qualitative studies, due to a focus on intervention effectiveness which qualitative research cannot answer. Instead, qualitative research is focussed on the experiences and perceptions of those involved in the programmes. This systematic review will address this gap by updating a 2009 review to examine qualitative studies. The aim is to generate deeper insight to help target resources which have the potential to save lives by preventing smoking initiation among children and young people.
\end{abstract}

Methods: This systematic review will be searching the following databases: the Cochrane Library, MEDLINE, EMBASE, PsycINFO, HMIC, ERIC, ASSIA, Web of Science and CINAHL. In order to identify additional references, we will consult the reference lists of a sample of systematic reviews and search relevant organizational websites in order to identify appropriate grey literature. The search strategy will include key words and database-specific subject headings relating to smoking, children and young people, health promotion and school. Authors will independently screen, assess data quality and extract data for synthesis. Study findings will be synthesised thematically using 'best-fit framework syntheses'. This allows for an existing set of themes to be used as a starting point to map or code included studies. These themes are then adapted as coding takes place to accommodate new emerging themes.

Discussion: This review will focus on qualitative studies that seek to examine the barriers and facilitators to the delivery of school-based smoking prevention programmes in order to inform the design of future theory-based interventions in schools to prevent children and young people from smoking.

Systematic review registration: PROSPERO CRD42014015483

Keywords: Children, Young people, Health promotion, Humans, Qualitative research, Schools, Smoking

\footnotetext{
* Correspondence: fiona.dobbie@stir.ac.uk

'Institute for Social Marketing, Faculty of Health Sciences and Sport,

University of Stirling, Stirling FK9 4LA, UK

Full list of author information is available at the end of the article
} 


\section{Background}

In the last two decades, much has been achieved to reduce smoking prevalence in both the child and adult population through tobacco control policies, particularly in high-income countries such as the UK. Findings from a Scottish school survey of adolescents aged 13-15 years called SALSUS (Scottish Schools Adolescent Lifestyle and Substance Use Survey) tracked a dramatic decline in adolescent's smoking over 20 years; $30 \%$ of boys and girls aged 15 were smoking regularly (defined as smoking at least one cigarette per week) in 1996 compared to just $7 \%$ in 2015 [1].

However, despite the recent decline in overall smoking prevalence in the UK [2], Hopkinson et al. [3] estimate that between 2010 and 2011, a total of 207,000 young people in the UK aged 11-15 started smoking. In addition, recent findings $(2013 / 2014)$ from the Health Behaviour of School-aged Children survey (which provides health and well-being data from young people, aged 11, 13 and 15, and from 43 countries across Europe and North America) found that on average, 22\% of boys and $13 \%$ of girls who had ever smoked a cigarette did so before the age of 13 or younger [4]. Prevalence of weekly smoking increases with age, with $1 \%$ of boys and $1 \%$ of girls aged 11 reporting weekly smoking. By the age of 13 , this increases to 4 and $3 \%$, and by the age of 15 , to $12 \%$ and $11 \%$, respectively [5].

Young people can become rapidly dependent on tobacco, and many smokers are addicted before they leave school, nearly $40 \%$ before the age of 16 [6]. Smokers who start at an early age tend to smoke more cigarettes per day in adulthood, smoke for longer, are less likely to quit, find it harder to quit and are more likely to die from a smoking-attributable cause [7-10]. Despite the health consequences of smoking generally developing in later life, there is evidence that young smokers can suffer lung function and lung growth impairment and at a greater risk to coughs and shortness of breath $[11,12]$. Therefore, youth smoking prevention interventions remain an important public health policy.

Over the last 30 years, schools have been particularly popular settings to deliver youth smoking prevention interventions [13]. The majority of children can be reached through school making this an obvious setting for smoking prevention interventions. However, evidence of the effectiveness of school-based prevention programmes is mixed, and outcomes vary by the type of programme delivered [13]. For example, a review by Flay found the most effective interventions were those that educated young people about social norms and peer influence on smoking behaviour, in comparison with those that just gave information on smoking harm [14]. In addition, previous systematic reviews of school-based smoking prevention programmes have tended to exclude qualitative studies, mainly because their focus is on intervention effectiveness $[13,15]$ which qualitative research cannot answer. A recent review of smoking interventions for young people used more inclusive criteria, including both qualitative and quantitative studies [16]; however, it had a specific focus on the equity impact of interventions and only included studies reporting smoking-related outcomes for two or more socioeconomic groups. There is, therefore, a gap in the systematic review evidence from qualitative studies [17], which focus on people's experiences and perceptions. Such studies can highlight important learning and generate deeper insight into the factors that contribute to the success or failure of school-based smoking prevention programmes.

In February 2010, the UK National Institute for Health and Care Excellence (NICE) published guidance on school-based interventions to prevent smoking [18]. As part of the evidence review to create these guidelines, a team of researchers conducted a systematic review of qualitative research published in 1990-2008 [19]. In 2013, NICE published an evidence update [20] which identified new studies and reviews, but did not systematically search for or include recent findings from qualitative studies.

This protocol is for a systematic review to update the existing review [19] with data from any new schoolbased programmes as well as any further contributions to the evidence for existing programmes (e.g. [21]).

\section{Review aim and research questions}

The aim of the review is to explore the facilitators and barriers to the delivery of school-based interventions to prevent smoking uptake in children and young people. The review will address the following research questions:

1. What factors aid the delivery of effective schoolbased interventions to prevent the uptake of smoking?

2. What are the barriers to successful delivery of effective school-based interventions to prevent the uptake of smoking?

\section{Methods}

This protocol has been designed using the PRISMA-P guidelines for systematic review protocol development [22] (see Additional file 1).

\section{Search strategy}

Three types of searching will be used. First, to replicate the 2009 review, the following electronic databases will be searched: Cochrane Library, MEDLINE, EMBASE, PsycINFO, HMIC, ERIC, ASSIA, Web of Science and 
CINAHL. Second, retrospective reference checking of a sample of systematic reviews and articles (e.g. by most recent publication type) will then be conducted by FD and KAn. Finally, website searching (using pre specified search terms) of key organisation and stakeholder groups (see Table 1) will be conducted to identify any unpublished literature. The search strategy (example provided in Table 2) will replicate the search strategy used in the original review with subject headings relating to smoking, children and young people, health promotion and school.

\section{Inclusion/exclusion criteria}

The Participants, Interventions, Comparisons and Outcomes (PICO) format [23] has been used to define the search strategy, to which $\mathrm{S}$ (setting), $\mathrm{T}$ (type of study) and $\mathrm{P}$ (type of publication) have been added. We chose

Table 1 Websites of key organisations to identify grey literature

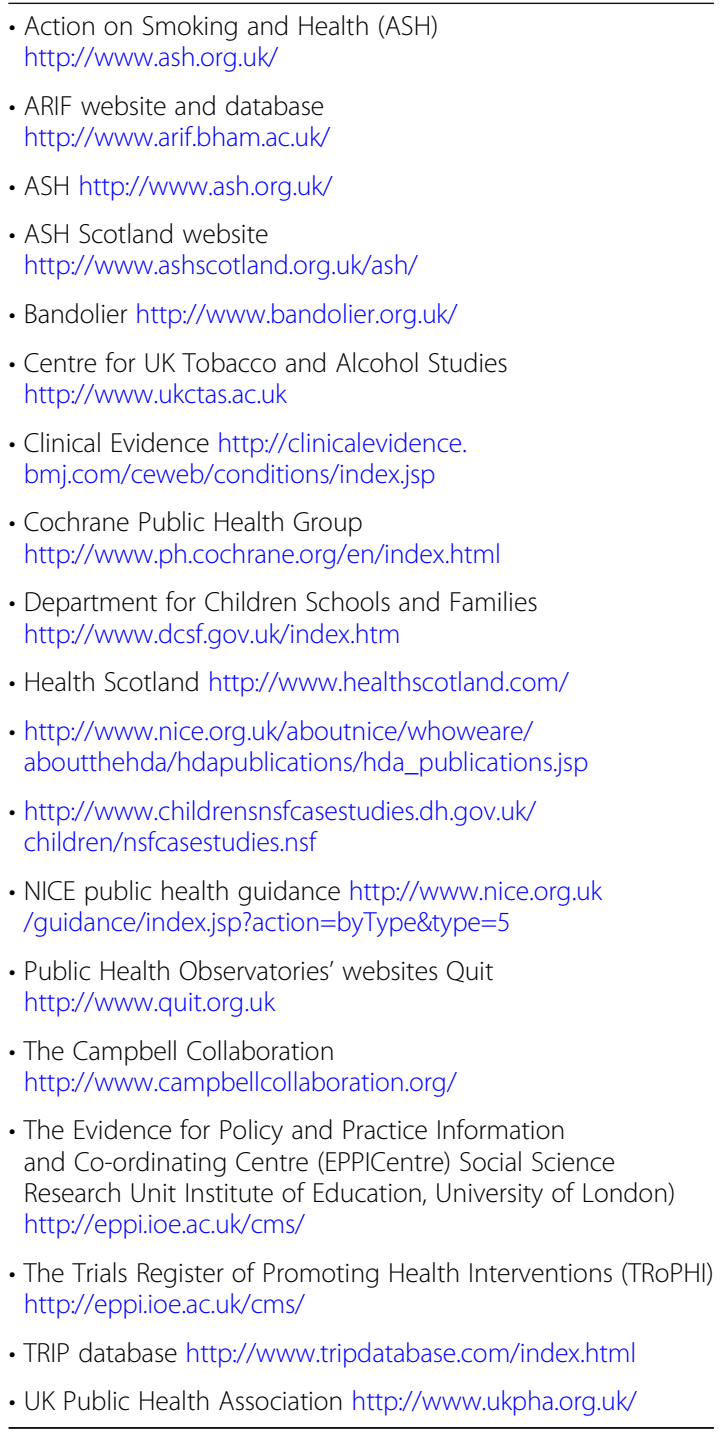

PICO over other tools (such as SPIDER and SPICE) because it appeared to be the most relevant and robust tool to identify the type of studies of interest. [24].

\section{Participants/population}

Participants are any child or young person attending primary or secondary school in a country of origin within the OECD (Organisation for Economic Co-operation and Development).

\section{Intervention(s) and exposure(s)}

The intervention will be any type of school-based smoking prevention intervention or programme. This could focus solely on smoking prevention or be included as part of a risk prevention programme (e.g. drugs, alcohol, sexual health). It will be made clear in the results section (of a future publication focusing on findings from the systematic review) which studies are smoking specific and which cover multiple risk factors. Only findings related to tobacco smoking will be included; marijuana smoking and electronic cigarettes will be excluded.

\section{Comparator(s)/control \\ Not applicable}

\section{Outcomes}

The outcome of this review is to explore the facilitators and barriers to the delivery of school-based interventions to prevent smoking uptake in children and young people.

\section{Setting}

All types of primary and secondary schools (e.g. state, public, special education, young offenders and faith schools) will be included.

\section{Types of study to be included}

Qualitative studies and mixed methods studies with a qualitative component. Pure quantitative studies or RCTs will be excluded (unless they have a process evaluation which includes a qualitative element). Studies will include any of the following qualitative methods: indepth, semi-structured, open interviews, group discussion, observation and ethnography.

\section{Publication characteristics}

Reference checking of a sample of recent systematic reviews (i.e. by relevant subject area and published within the last 5 years), journal articles and grey literature published in English between 2008 and 2017 will be eligible for review, using pre-specified search terms. Conference abstracts will be excluded due to a lack of data, although a search will be made for a full-text paper. 
Table 2 MEDLINE example full search strategy. This search strategy will be adapted for each database

\begin{tabular}{|c|c|c|}
\hline $\begin{array}{l}\text { 1. young people.mp. } \\
\text { 2. young person\$.mp. } \\
\text { 3. young adult\$.mp. } \\
\text { 4. adolescent\$.mp. } \\
\text { 5. youth\$.mp. } \\
\text { 6. teenage\$.mp. } \\
\text { 7. girl\$.mp. } \\
\text { 8. boy\$.mp. } \\
\text { 9. exp Adolescent/ } \\
\text { 10. Child/ } \\
\text { 11. child\$.mp. } \\
\text { 12. or/1-11 } \\
\text { 13. exp Schools/ } \\
\text { 14. academy.mp. } \\
\text { 15. academies.mp. } \\
\text { 16. city } \\
\text { technology.mp. } \\
\text { 17. education } \\
\text { centre\$.mp. }\end{array}$ & $\begin{array}{l}\text { 18. secure unit\$.mp. } \\
\text { 19. training unit\$.mp. } \\
\text { 20. secure training.mp. } \\
\text { 21. referral unit\$.mp. } \\
\text { 22. school\$.mp. } \\
\text { 23. (offender\$ adj institute\$).mp. } \\
\text { 24. further education.mp. } \\
\text { 25. or/13-25 } \\
\text { 26. } 25 \text { and } 12 \\
\text { 27. health promotion.mp. or exp Health Promotion/ } \\
\text { 28. health education.mp. or exp Health Education/ } \\
\text { 29. primary prevention.mp. or exp Primary Prevention/ } \\
\text { 30. (campaign or teach\$ or advis } \$ \text { or counsel\$ or promot } \$ \text { or } \\
\text { encourag\$).mp. }\end{array}$ & 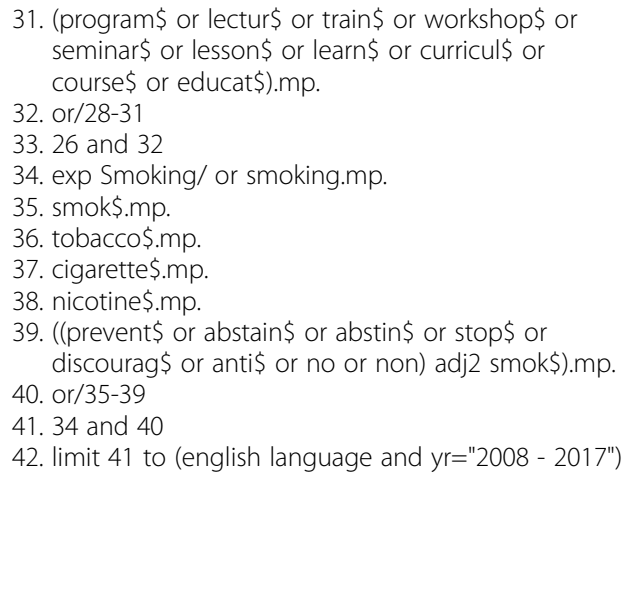 \\
\hline
\end{tabular}

\section{Data management}

Once the search terms have been piloted and finalised, electronic databases will be searched and references exported to RefWorks (ProQuest LLC) bibliographic software for storage and removal of duplicates. After removing duplicates, title and abstracts will be reviewed to identify relevant studies using a pre-defined checklist based on the inclusion/exclusion criteria described in the previous section. FD will screen all references, with second review shared by three other members of the research team (KAl, HL, KAn). Full papers will be retrieved for studies deemed potentially relevant. Double screening of full papers will be conducted (FD, $\mathrm{KAl}$ and $\mathrm{KAn}$ ), and those deemed irrelevant will be removed. Where two reviewers disagree, a third reviewer will screen the full paper for inclusion or exclusion. This will then generate a final list of studies for full review. At this stage, the other elements of the search method will be conducted (website review and reference checking).

\section{Quality assessment}

All studies (including grey literature) that meet the inclusion criteria for full review will be independently reviewed by $\mathrm{FD}, \mathrm{KAl}$ and $\mathrm{KAn}$. In line with the previous review, the critical appraisal checklist developed by NICE for qualitative studies will be used to review the selected articles [25]. NICE provides evidence-based national guidance and advice to improve health and social care, predominantly in England but also to the rest of the UK. It is sponsored by the Department of Health. For each qualitative study, the tool will assess 14 items under the following domains: the theoretical approach and clarity of its aims; the rigour of the methods; how well the data collection was carried out; the relationship between the researcher and participants and reliability of the methods; the richness of the data and rigour and reliability of the analysis and findings; and the reporting of ethical issues. An overall assessment of the study's relevance and one of three final gradings will be given according to how many of the checklist criteria have been fulfilled, and if not fulfilled, whether the conclusions are likely to alter or not. A grading of ' ++' will be made if all or most of the checklist criteria have been fulfilled, where they have not been fulfilled, the conclusions are very unlikely to alter; ' + ' if some of the checklist criteria have been fulfilled, where they have not been fulfilled, or not adequately described, the conclusions are unlikely to alter; and '-' if few or no checklist criteria have been fulfilled and the conclusions are likely or very likely to alter [25].

\section{Strategy for data collection and synthesis}

Data will be extracted by FD and KAl using a 'data extraction template' (created using Microsoft Word and piloted with two articles first) that will record the following: aims of research, sample, country where research was conducted, research design and key findings (of relevance to the particular aims of this review only, and quality score from critical review). Study findings will be synthesised thematically using best-fit framework synthesis [26]. This allows for an existing set of themes to be used as a starting point to map or code included studies. These themes are then adapted as coding takes place to accommodate new emerging themes. This approach is particularly useful for the proposed systematic review in three ways. First, it enables utilisation of the themes identified in the previous systematic review [19]. Second, it is an efficient and pragmatic approach to coding when timescales are limited. Third, the framework approach involves 'charting' of data in a matrix which allows for greater transparency of coding and, thus, supports coding and analysis via a team of researchers [27]. Our synthesis will be mindful of 'dissonant voices' and note themes that are discussed from a range of different 
perspectives. For example, using peer educators to deliver smoking prevention messages may be positive in terms of reach and credibility but could also be problematic if they are not conveying accurate messages. Once data is extracted, it will be examined for similarities and dissonance. It is likely that, initially, findings will be grouped by setting (primary or secondary school) and by population (pupils, teaching staff, other staff, parents). The review will be reported with reference to the ENTREQ (ENhancing TRansparency in the REporting of Qualitative health research) statement [28].

\section{Discussion}

Much has been written about the effectiveness of school-based interventions to prevent children and young people from smoking, with mixed results depending on the type of programme delivered [13, 14, 29, 30]. However, little is known about the factors that influence their effectiveness. This review will focus on qualitative studies that seek to address this gap by examining the barriers and facilitators to the delivery of school-based smoking prevention programmes. This will generate greater insight to inform the design of future theorybased design interventions in schools to prevent children and young people from smoking. This could help target resources appropriately and has the potential to save lives by preventing smoking initiation among children and young people.

There are some limitations to the outlined systematic review. The restriction to English is acknowledged as a language bias. The cost of high-quality translations of in-depth qualitative data are beyond the resources of this review; however, non-English language studies identified at the screening stages and excluded from the synthesis will be listed in an appendix to the review to aid future reviewers.

\section{Additional file}

Additional file 1: PRISMA-P checklist. (DOCX 29 kb)

\begin{abstract}
Abbreviations
ASSIA: Applied Social Science Index and Abstracts; CINAHL: Cumulative Index to Nursing and Allied Health Literature; ENTREQ: ENhancing Transparency in the REporting of Qualitative health research; ERIC: Education Resources Information Center; HMIC: Health Management Information Consortium; NICE: National Institute for Health and Care Excellence; OECD: Organisation for Economic Co-operation and Development; PICO: Participants, Interventions, Comparisons and Outcomes; PRISMA-P: Preferred Reporting Items for Systematic review and Meta-analysis Protocols; RCTs: Randomised controlled trials; SALSUS: Scottish Schools Adolescent Lifestyle and Substance Use Survey; SPICE: Setting, Perspective, Intervention, Comparison, Evaluation; SPIDER: Sample, Phenomenon of Interest, Design, Evaluation, Research type
\end{abstract}

\section{Acknowledgements}

The authors wish to thank Professor James Thomas and Dr. Heather Wardle for commenting on the draft protocol.

\section{Funding}

The systematic review is being conducted as part of Fiona Dobbie's part-time $\mathrm{PhD}$ which is funded by the University of Stirling. The Institute for Social Marketing is a member of the UK Centre for Tobacco and Alcohol Studies (http://ukctas.net/). Funding for UKCTAS from the British Heart Foundation, Cancer Research UK, the Economic and Social Research Council, the Medical Research Council and the National Institute of Health Research, under the auspices of the UK Clinical Research Collaboration, is gratefully acknowledged. The funders had no role in the study design, data collection and analysis, decision to publish or preparation of the manuscript.

\section{Availability of data and materials}

Not applicable

\section{Note}

This systematic review is being conducted as part of Fiona Dobbie's part-time PhD, funded by the University of Stirling. She is the guarantor of the review.

\section{Authors' contributions}

FD and $L B$ designed the original PhD study proposal. FD drafted the protocol, with contributions from KAn, WW, LB, AA, SW, KAl and HL. FD, KAn and $L B$ defined the search terms and inclusion and exclusion criteria. All authors read and approved the final manuscript.

Ethics approval and consent to participate

Not applicable

\section{Consent for publication}

Not applicable

\section{Competing interests}

The authors declare that they have no competing interests.

\section{Publisher's Note}

Springer Nature remains neutral with regard to jurisdictional claims in published maps and institutional affiliations.

\section{Author details}

${ }^{1}$ Institute for Social Marketing, Faculty of Health Sciences and Sport, University of Stirling, Stirling FK9 4LA, UK. ${ }^{2}$ DECIPHer (Centre for the Development and Evaluation of Complex Public Health Interventions), Cardiff University, 1-3, Museum Place, Cardiff CF10 3AT, UK. ${ }^{3}$ University of Stirling, Stirling FK9 4LA, UK. ${ }^{4}$ Usher Institute of Population Health Sciences and Informatics, University of Edinburgh, Edinburgh EH8 9AG, UK. ${ }^{5}$ Faculty of Health Sciences and Sport, University of Stirling, Stirling FK9 4LA, UK.

Received: 4 August 2017 Accepted: 20 March 2018

Published online: 06 April 2018

\section{References}

1. Scottish Government. Scottish Schools Adolescent Lifestyle and Substance Use Survey (SALSUS) smoking report (2015). Ipsos MORI Scotland for the Scottish Government. 2016. http://www.gov.scot/Resource/0050/00508401. pdf. Accessed 16 Jun 2017.

2. Fuller E, Hawkins V. Smoking drinking and drug use among young people in England in 2013. London: The Information Centre for Health and Social Care; 2014. Available http://www.content.digital.nhs.uk/catalogue/ PUB14579/smok-drin-drug-youn-peop-eng-2013-rep.pdf Accessed 22 Jul 2014.

3. Hopkinson NS, Lester-George A, Ormiston-Smith N, Cox A, Arnott D. Child uptake of smoking by area across the UK. Thorax. 2014;69:8735. https://doi.org/10.1136/thoraxjnl-2013-204379.

4. Currie C. Social determinants of health and well-being among young people: Health Behaviour in School-aged Children (HBSC) study: international report from the 2009/2010 survey. Copenhagen: World Health Organization, Regional Office for Europe; 2012.

5. Inchley J, Currie D, Young T, Samdal O, Torsheim T, Augustson L, Mathison F, Aleman-Diaz A, Molcho M, Weber M, Barnekow V. Growing up unequal. HBSC 2016 study (2013/2014 survey). Health policy for children and adolescents no. 7. Copenhagen: WHO Regional Office for Europe; 2016. 
Available http://www.euro.who.int/_data/assets/pdf_file/0003/303438/ HSBC-No.7-Growing-up-unequal-Full-Report.pdf?ua=1 Accessed 2 May 2017.

6. Dunstan S, Robinson S. General lifestyle survey overview: a report on the 2010 general lifestyle survey. London: Office for National Statistics; 2012. http://www.Ons.Gov.uk/ons/rel/ghs/general-lifestylesurvey/2010/index.html Accessed 22 Jul 2014

7. Department of Health. Smoking kills: a white paper on tobacco. London: Department of Health; 1998.

8. Difranza JR, Richmond JB. Let the children be heard: lessons from studies of the early onset of tobacco addiction. Pediatrics. 2008;121(3):623-4. https://doi.org/10.1542/peds.2007-3696.

9. Stanton A, Grimshaw G. Tobacco cessation interventions for young people. Cochrane Database Syst Rev. 2013:CD003289. https://doi.org/10.1002/ 14651858.CD003289.pub5.

10. Tyas SL, Pederson LL. Psychosocial factors related to adolescent smoking: a critical review of the literature. Tob Control. 1998;7:409-20.

11. Health and Social Care Information Centre (HSCIC) Lifestyles Statistics. Statistics on smoking, England, 2012. 2012: https://catalogue.ic.nhs.uk/ publications/publichealth/smoking/smok-eng-2012/smok-eng-2012-rep.pdf. Accessed 14 Feb 2013.

12. Royal College of Physicians. Smoking and the young: a report of a working party of the Royal College of Physicians. London: Royal College of Physicians; 1992.

13. Thomas RE, McLellan J, Perera R. School-based programmes for preventing smoking. Evid-Based Child Health. 2013;8:1616-2040. https://doi.org/10.1002/ebch.1937.

14. Flay BR. The long-term promise of effective school-based smoking prevention programs. In: Bonnie RJ, Stratton K, Wallace RB, editors. Ending the tobacco problem: a blueprint for the nation. Committee on Reducing Tobacco Use: strategies, barriers, and consequences board on population health and public health practice. Washington, DD: Institute Of Medicine of The National Academies, The National Academies Press; 2007. p. 449-77.

15. Mercken L, Moore L, Crone MR, De Vries H, De Bourdeaudhuij I, Lien N, Fagiano F, Vitória PD, Van Lenthe FJ. The effectiveness of school-based smoking prevention interventions among low- and high-SES European teenagers. Health Educ Res. 2012;27(3):459-469. https://doi.org/10.1093/her/cys017.

16. Brown T, Platt $S$, Amos A. Equity impact of interventions and policies to reduce smoking in youth: systematic review. Tob Control. 2014;23(e2):e98105. https://doi.org/10.1136/tobaccocontrol-2013-051451.

17. Thomas J, Harden A. Methods for the thematic synthesis of qualitative research in systematic reviews. BMC Med Res Methodol. 2008;8(45):1-10. ISSN 1471-2288

18. NICE. Smoking prevention in schools. Public health guideline PH23. 2010. Available http://guidance.nice.org.uk/PH23 Accessed 7 Jun 2014.

19. Bauld L, Brandling T, Templeton L. Facilitators and barriers to the delivery of school-based interventions to prevent the uptake of smoking among children: a systematic review of qualitative research. 2009. https://www.nice. org.uk/guidance/ph23/evidence/review-of-qualitative-literature-pdf371532061. Accessed 21 Jun 2017.

20. NICE. School-based interventions to prevent the uptake of smoking among children and young people. Evidence Update 38. April 2013. Available http://www.evidence.nhs.uk/evidence-update-38 Accessed 10 Dec 2014.

21. Holliday J, Audrey S, Moore L, Parry-Langdon N, Campbell R. High fidelity? How should we consider variations in the delivery of school-based health promotion interventions? Health Educ J. 2009;68(1):44-62.

22. Moher D, Shamseer L, Clarke M, Ghersi D, Liberati A, Petticrew M, Shekelle P, Stewart LA, PRISMA-P Group. Preferred reporting items for systematic review and meta-analysis protocols (PRISMA-P) 2015 statement. Syst Rev. 2015:4:1. https://doi.org/10.1186/2046-4053-4-1.

23. Higgins JPT, Green S. Cochrane handbook for systematic reviews of interventions version 5.1.0. The Cochrane Collaboration; 2011. Available from http://handbook.cochrane.org Accessed 22 Jul 2014.

24. Methley AM, Campbell S, Chew-Graham C, McNally R, Cheraghi-Sohi S PICO, PICOS and SPIDER: a comparison study of PICO, PICOS and SPIDER: a comparison study of qualitative systematic reviews. BMC Health Serv Res. 2014;14:579. https://doi.org/10.1186/s12913-014-0579-0

25. NICE. Appendix H Quality appraisal checklist-qualitative studies. In: NICE. Methods for the development of NICE public health guidance. Process and methods [PMG4]. $3^{\text {rd }}$ edition. 2012. Available https://www.nice.org.uk/ process/pmg4/chapter/appendix-h-quality-appraisal-checklist-qualitativestudies Accessed 2 May 2017.
26. Carroll C, Booth A, Cooper K. A worked example of "best fit" framework synthesis: a systematic review of views concerning the taking of some potential chemopreventive agents. BMC Med Res Methodol. 2011;11:29. https://doi.org/10.1186/1471-2288-11-29.

27. Dixon-Woods M. Using framework-based synthesis for conducting reviews of qualitative studies. BMC Med. 2011;9:39. https://doi.org/10.1186/1741-7015-9-39.

28. Tong A, Flemming K, Mclnnes E, Oliver S, Craig J. Enhancing transparency in reporting the synthesis of qualitative research: ENTREQ. BMC Med Res Methodol. 2012;12:181. https://doi.org/10.1186/1471-2288-12-181.

29. Flay BR. Effectiveness of school-based smoking prevention programs. Prepared for the World Health Organization tobacco free, initiative, January 31st, 2008.

30. Fletcher A, Bonell C, Hargreaves J. School effects on young people's drug use: a systematic review of intervention and observational studies. J Adolesc Health. 2008;42(3):209-20.

\section{Submit your next manuscript to BioMed Central and we will help you at every step:}

- We accept pre-submission inquiries

- Our selector tool helps you to find the most relevant journal

- We provide round the clock customer support

- Convenient online submission

- Thorough peer review

- Inclusion in PubMed and all major indexing services

- Maximum visibility for your research

Submit your manuscript at www.biomedcentral.com/submit
Biomed Central 